\title{
Complete augmentation of diffuse narrowing of the aorta with Williams syndrome by using an overturn approach
}

Masaaki Yamagishi, MD, Keisuke Shuntoh, MD, Tsutomu Matsushita, MD, Katsuji Fujiwara, MD, Takeshi Shinkawa, MD, Takako Miyazaki, MD, and Nobuo Kitamura, MD, Kyoto, Japan

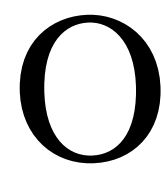
ne-staged complete augmentation of diffuse narrowing of the aorta extending from the ascending aorta (AAo) to the descending aorta (DAo) with Williams syndrome $^{1}$ is extremely difficult ${ }^{2}$ because of the lack of a good surgical approach. For the treatment of diffuse aortic stenosis, we report an innovative surgical technique involving unifocalization of the neck branch arteries ${ }^{3}$ and complete aortic augmentation by using an overturn approach.

\section{Clinical Summary}

A male infant weighing $7400 \mathrm{~g}$ had an elfin face. Diagnosis of Williams syndrome was confirmed on the basis of a microscopic deletion in the chromosome 7q11.23 region. Cardiac catheterization demonstrated diffuse narrowing of the aorta extending from the AAo to the DAo. Neck arteries branched from the aorta with one trunk and further branched into 3 arteries with narrowed orifices. The pressure gradient between the AAo and the left ventricle was $100 \mathrm{~mm} \mathrm{Hg}$. The aortic valve was competent without stenosis. Segmental stenosis of the pulmonary bifurcation was also detected.

The operation was performed through a median sternotomy when the infant was 10 months old. The neck arterial branches were dissected as distally as possible. Arterial cannulas were inserted into the AAo, the brachiocephalic trunk, and the left common carotid artery to ensure continuous cerebral and coronary perfusion. Another arterial cannula for lower-body perfusion was directly inserted into the DAo just above the diaphragm. After venous cannulas were inserted into both venae cavae, moderate hypothermic cardiopulmonary bypass was established. Throughout the operation, circulation was never arrested. The AAo and the proximal DAo were clamped, and neck arteries were squeezed by using a thin tape with a tourniquet. While the heart was beating, the origins of the neck arteries were transected, and the degenerated thick walls of each stump were completely resected (Figure 1, $A$ ). Unifocalization of the neck arteries by means of anastomosis of adjacent neck arteries in a side-to-side fashion was accomplished,

From the Department of Pediatric Cardiovascular Surgery, Children's Research Hospital, Kyoto Prefectural University of Medicine, Kyoto, Japan.

Received for publication May 30, 2002; accepted for publication June 13, 2002.

Address for reprints: Masaaki Yamagishi, MD, Department of Pediatric Cardiovascular Surgery, Children's Research Hospital, Kyoto Prefectural University of Medicine, Kawaramachi, Hirokoji, Kamikyo-ku, Kyoto, 6028566 Japan (E-mail: myama@koto.kpu-m.ac.jp).

J Thorac Cardiovasc Surg 2003;125:1556-8

Copyright $\odot 2003$ by The American Association for Thoracic Surgery $0022-5223 / 2003 \$ 30.00+0$

doi:10.1016/S0022-5223(03)00008-4
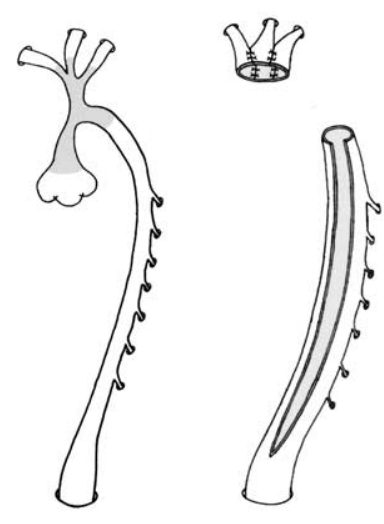

$A$

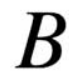

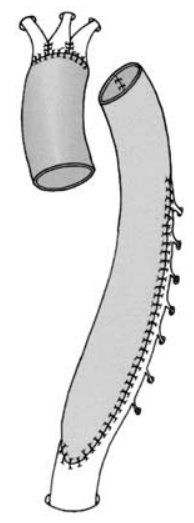

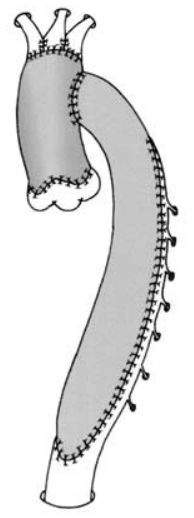

$D$

Figure 1. Schematic representation of the operative procedure. $A$, Resected areas at the $A A_{0}$ and the origin of the neck arteries are indicated by the gray zone. $B$, Distal stumps of the neck arteries were unifocalized. The anterior wall of the DAo was incised longitudinally. $C$, The homograft was anastomosed to the unifocalized neck arteries in an end-to-end fashion. The DAo was augmented with another homograft patch. $D$, The AAo homograft was anastomosed to the proximal AAo stump. The cranial end of the DAo homograft was anastomosed to the left side wall of the AAo homograft in an end-to-side fashion.

as we previously reported (Figure $1, B) .^{3}$ The newly created common orifice of the unifocalized neck arteries was anastomosed with a homograft in an end-to-end fashion. The heart was then arrested, and the AAo cannula was withdrawn. The DAo was clamped only on the cranial side of the DAo cannula. The proximal end of the DAo was separated from the aortic arch. For the overturn approach, the following sites were incised: the right pulmonary artery was transected distal to the stenotic lesion; the superior and inferior vena cavae were transected just before the junction with the right atrium; and the left atrial wall around the right pulmonary venous orifices was resected. Leaving the continuity of the main pulmonary trunk and the left pulmonary veins, the heart was overturned leftward (Figure 2, A). A wide field of view of the posterior mediastinal space was opened without any hindrance. The posterior mediastinum was opened longitudinally, and the anterior surface of the narrowed DAo was totally unshrouded. The posterior aspect of the DAo was not dissected to preserve the intercostal arteries. The anterior wall of the narrowed DAo was cut through longitudinally (Figure 1, B). A trimmed homograft patch $30 \mathrm{~mm}$ in width was anastomosed with the opening of the DAo (Figure 1, $C$, and Figure 2, B). The cranial end 

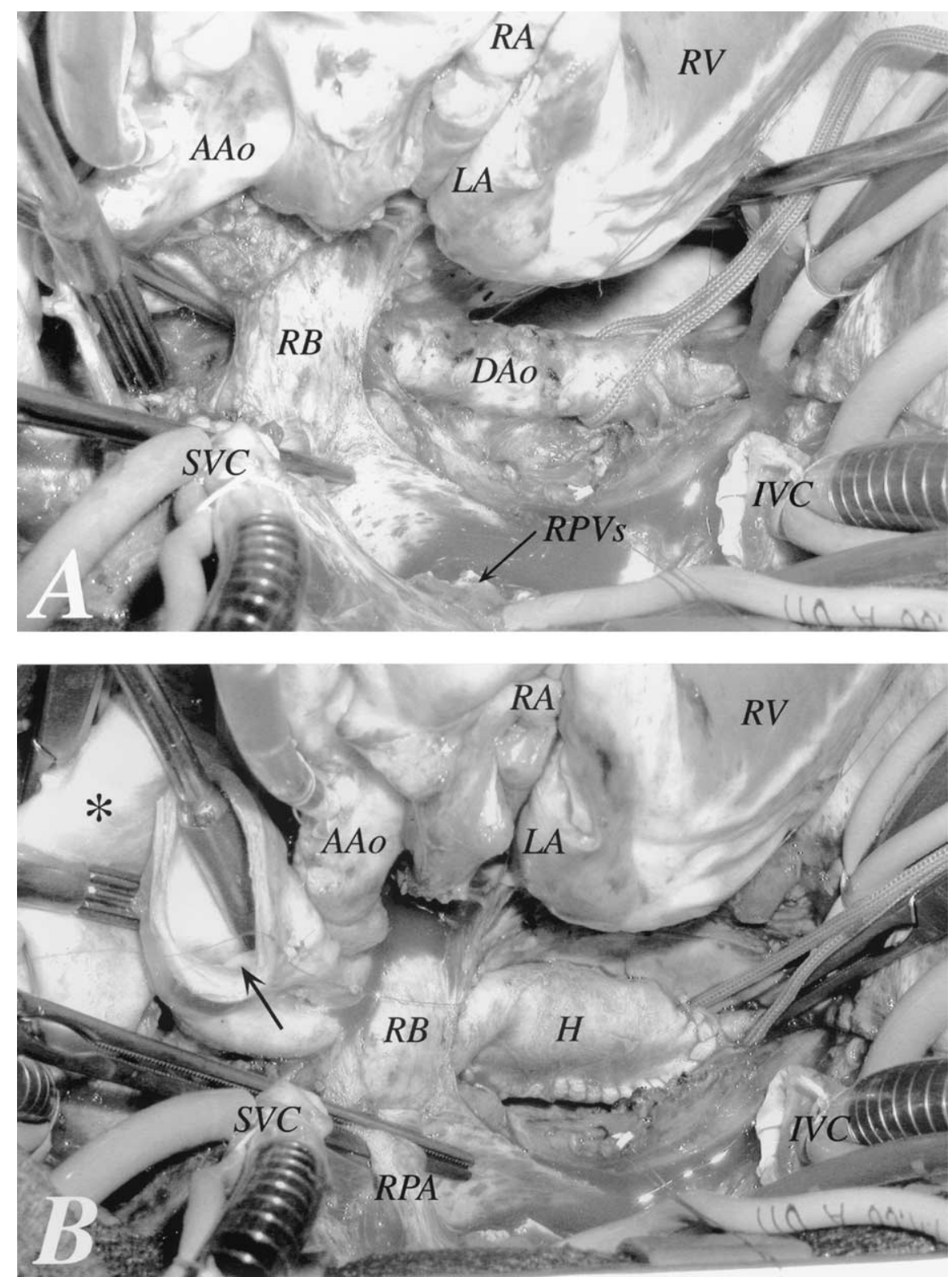

Figure 2. Intraoperative findings. $A$, Findings after the caval veins, the right pulmonary artery, and the left atrial wall, including the right pulmonary venous orifices, were incised and then the heart was turned over leftward are shown. A wide-field of view of the posterior mediastinal space was opened. $B$, Posterior to the right bronchus, a homograft patch was anastomosed to the longitudinal incision of the DAo. The cranial end of the homograft was sewn in a roll shape (arrow). The asterisk indicates the AAo homograft. $H$, Homograft patch; IVC, inferior vena cava; $L A$, left atrium; $R A$, right atrium; $R B$, right bronchus; $R P V s$, right pulmonary veins; $R V$, right ventricle.

of the homograft was sewn in a roll shape. After completion of the DAo augmentation, the heart was turned back, and the left atrial wall was restored. The continuity of the right pulmonary artery was reconstructed, and the stenotic pulmonary bifurcation was augmented by using an autologous pericardial patch. After the AAo, including the supravalvular ridge, was totally resected, longitudinal incisions a few millimeters in length were made at each sinus of Valsalva. The end opposite to the unifocalized neck arteries of the homograft was anastomosed with the proximal stump of the AAo in an end-to-end fashion. Finally, the cranial end of the roll-shaped DA homograft was anastomosed to the left side wall of the AAo homograft in an end-to-side fashion (Figure 1, D). After both vena cavae were restored and the cardiopulmonary bypass was disconnected, the hemodynamics were good. Postoperative magnetic resonance angiography showed well-augmented thoracic aorta and neck arteries without residual stenosis. 


\section{Discussion}

Complete relief of aortic narrowing in Williams syndrome is essential to eliminate residual left ventricular pressure overload. However, 1-staged complete augmentation of the diffuse narrowing of thoracic aorta with Williams syndrome is impossible by means of conventional surgical approaches and techniques. By using the innovative overturn approach, a holoscopic operative field of the DAo was opened. Complete augmentation of the aorta with diffuse narrowing can be accomplished in conjunction with the technique of Brom ${ }^{4}$ for supravalvular aortic stenosis and the unifocalization technique ${ }^{3}$ for narrowing of the proximal neck arteries. By using this combined technique, residual stenosis and late restenosis might be successfully avoided. The overturn approach is applicable to other operations in the posterior mediasti- num, such as unifocalization of the major aortopulmonary collateral arteries.

\section{References}

1. Radford DJ, Pohlner PG. The middle aortic syndrome: an important feature of Williams' syndrome. Cardiol Young. 2000;10:597-602.

2. Brown JW, Ruzmetov M, Vijay P, Turrentine MW. Surgical repair of congenital supravalvular aortic stenosis in children. Eur J Cardiothorac Surg. 2002;21:50-6.

3. Yamada Y, Yamagishi M, Shuntoh K, Okano T, Hayashida K, Shinkawa T, et al. Unifocalization of the neck arteries combined with aortic arch replacement for Williams syndrome. J Thorac Cardiovasc Surg. 2002;123:579-80.

4. Brom AG. Obstruction of the left ventricular outflow tract. In: Khonsari $\mathrm{S}$, editor. Cardiac surgery: safeguards and pitfalls in operative technique. Rockville (MD): Aspen; 1988. p. 276-80. 